\title{
Application of Bearing Diagnosis Based on Wavelet Preprocessing Neural Network
}

\author{
Chen Jiang-bo ${ }^{1, a^{*}}$, Zeng Shi-xian ${ }^{1, b}$ and Li Yan ${ }^{1, c}$ \\ ${ }^{1}$ Qingdao Huanghai University, Qingdao, Shandong, China \\ ajiangbochen1981@163.com, ' liyanvsl@126.com
}

Keywords: Wavelet preprocessing, Neural network, Parameter modification, Fault diagnosis, Convergence

\begin{abstract}
According to compactly supported characteristics of bearing local damage diagnosis based on wavelet analysis, the construction of compactly supported orthogonal function method are studied. With the wavelet multi-resolution analysis and multivariate time series analysis as the foundation, the parameters of weights, scaling and translation in the model are modified. Based on MATLAB, the non-stationary signal of fault bearing acceleration wave is numerical simulated and calculated. Through the comparison of convergence between the parameters revising before and after, it shows that the error on the amplitude and the convergence efficiency of parameter modification are better than the fixed before, which can significantly improve the accuracy of bearing fault diagnosis and signal processing capacity.
\end{abstract}

\section{Introduction}

Bearing as one of the most widely used parts in rotating machinery, its working condition for the stability of the mechanical equipment. In actual production, bearing fault diagnosis accuracy and real-time performance for reducing the sudden interruption of mechanical equipment, reduce the economic loss and accident is of great significance[1] .

The barrier of diagnostic techniques such as time domain and frequency domain method has been widely applied at present[2], the main through the vibration characteristics of bearing under different damage fault analysis and prediction, has a good visual, but for the local pitting, micro cracks and other damage, due to the impact time is shorter, the conventional fault detection technology cannot achieve the desired effect. Aiming at this problem, this paper puts forward the application of neural network based on wavelet pretreatment technology, through the construction of mathematical model and parameter adjustment, improve the bearing fault signal analysis and processing ability, and adopt the method of numerical simulation verify its feasibility.

\section{The mechanism of rolling bearing fault diagnosis}

The fault characteristic frequency: Rolling bearings are the major factors of early defect or damage, improper material defects, manufacturing technology, poor lubrication, etc, after a period of time to adjust,It easy to appear fatigue spalling, the phenomenon such as crack, affect the normal work of the mechanical equipment. Different bearing failure will produce different periodic pulse according to the resonant frequency of the signal acquisition and processing, and combined with the corresponding data analysis technology, able to diagnose the bearing fault [3]. In the work, bearing inner ring failure frequency is:

$$
f_{i p}=N \frac{\sin \alpha}{\sin \alpha+\sin \beta}\left|f_{o}-f_{i}\right|
$$

Bearing outer ring failure frequency is:

$$
f_{o p}=N \frac{\sin \beta}{\sin \alpha+\sin \beta}\left|f_{o}-f_{i}\right|
$$

The rotation of the roller, failure frequency is: 


$$
f_{b c}=\frac{2 \cos \alpha \sin \alpha \sin \beta}{\sin ^{2} \alpha-\sin ^{2} \beta}\left|f_{o}-f_{i}\right|
$$

According to the formula (1) (3), $\alpha$ for the ball and raceway contact Angle, $\beta$ for the contact Angle of ball bearing with inner raceway, $N$ is the number of ball bearing, $f_{i}$ and $f_{o}$ are the rotation frequency of the bearing inner and outer ring respectively.

The diagnosis process: Because of the bearing fault frequency has a wide frequency domain, It needs to be a spectrum of high frequency vibration mode as the main research object, which is separated through the signal acquisition and bandpass filter failure frequency. Through the wavelet pretreatment, signal-to-noise ratio (SNR) can efficiently realize the fault signal processing [4]. In order to eliminate the fault signal of high frequency attenuation components, get direct expression fault information of low frequency envelope signal,we can use the Hilbert transform, demodulate envelope analysis object. under the application of the neural network technology, the multiresolution signal characteristic vector can be efficiently obtained and make the process as shown in Fig.1.

From the point of view of signal filtering, building a tight branch of orthogonal wavelet can better complete the signal samples of every point. After the high and low frequency signal decomposition, We can size classification, according to the energy scale under different feature vector.

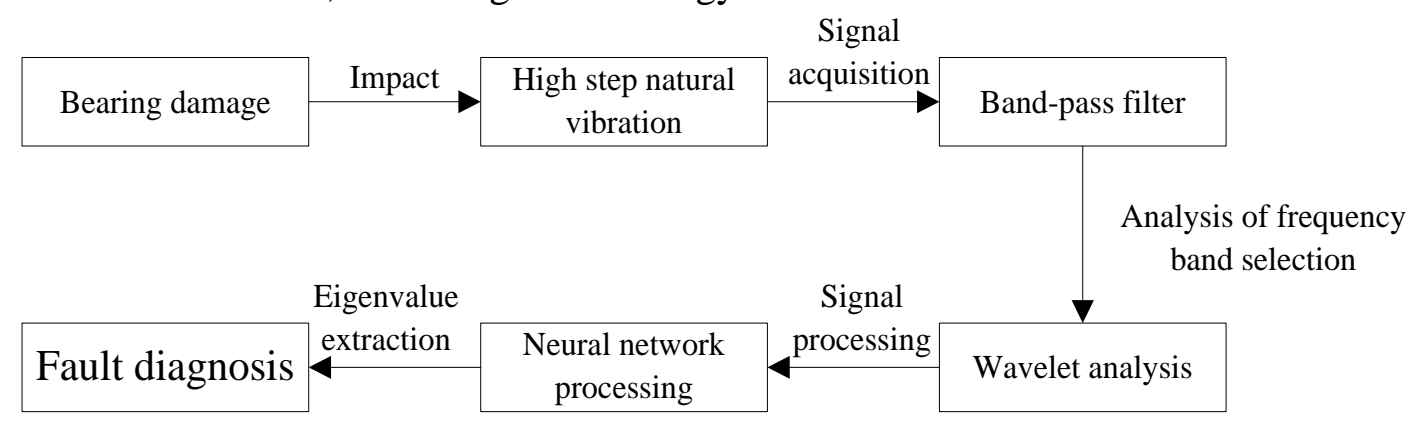

Fig. 1 fault diagnosis process

Construction of compactly supported wavelets: Wavelet analysis is based on the traditional signal analysis, which have the characteristics of tight branch, the signal-to-noise ratio (SNR) is widely used in separation and weak signal extraction. The methods of wavelet analysis and processing method are short-time Fourier transform, wavelet transform, Randon - Wigner transform, time-frequency analysis etc.

For analog signal $f(t)$, the wavelet transform result $W_{f}(a, b)$ can be represented as:

$$
W_{f}(a, b)=\int_{R} f(t) \varphi_{a b}(t) d t
$$

According to the formula (4), $\varphi_{a b}(t)$ is the result of the wavelet basis function $\varphi(t)$. Due to the partial bearing damage signal belongs to the typical non-stationary signal, which has strong discreteness in frequency detection, in this paper,using the construction of compactly supported wavelet improve the quality of signal processing, $\varphi(x)$ is the orthogonal scaling function.

When building compactly supported wavelets, It need to satisfy the following conditions:

(1) $\{\varphi(x-k)\}_{k \in z}$ is a standard orthogonality, meet the double scale equation $\varphi(x / 2)=\sum_{n \in Z} h_{k} \varphi(x-n)$;

(2) It need meet $\int \varphi(x) d x \neq 0$, and $\varphi(x)$ is compactly supported function. According to the support of the wavelet component conditions ,the function can be deduced:

$$
m_{\varphi}(0)=1, \quad\left|m_{\varphi}(\xi)\right|^{2}+\left|m_{\varphi}(\xi+\pi)\right|^{2}=1, \quad \hat{\varphi(\xi)}=\frac{1}{\sqrt{2 \pi}} \prod_{j=1}^{\infty} m_{\varphi}\left(2^{-j} \xi\right)
$$

$m_{\varphi}(\xi)$ is composed of two dimensions equations of frequency domain equations, the relationship satisfaction is $m_{\varphi}(\xi)=\hat{\varphi}(2 \xi) / \hat{\varphi}(\xi)$. 
Set $\ell(\xi)$ as trigonometric polynomial, $N$ for natural Numbers, compactly supported wavelet function is obtained through the following ways:

$$
\begin{aligned}
& m_{\varphi}(\xi)=\left(\frac{1+e^{-i \xi}}{2}\right)^{N} \ell(\xi) \\
& P\left(e^{-i \xi}\right)=e^{-i N \xi} M(\xi) \\
& M(\xi)=\sum_{n=0}^{N} a_{\mathrm{n}} \cos (n \xi) \\
& \left|m_{\varphi}(\xi)\right|^{2}=\mathrm{M}_{\varphi}(\xi)=\left(\cos ^{2} \frac{\xi}{2}\right)^{N} P\left(\sin ^{2} \frac{\xi}{2}\right)
\end{aligned}
$$

\section{The construction of wavelet neural network}

The wavelet neural network structure: Preprocessing based on wavelet neural network technology in the signal processing of bearing has good application effect. wavelet transform can carry on the specific to the target signal multi-scale scaling and translation, the parameters through the network, can make the model structure is more simple, faster convergence speed of computing.

Wavelet neural network in structure includes loose and intelligent, it can adjust multi-scale signals by scaling and translation, can effectively extract local information from the fault signal , the network structure keep the linear relationship between basis function and network weights, which improves the convergence in the local optimization calculation. Under the action of the wavelet basis function, Specific neural network parameters can solve the blindness of conventional neural network algorithm.

In preprocessing based on wavelet neural network based on dimension,it can be divided into one dimensional and multidimensional network. Among them, the one-dimensional only to transform a one-dimensional wavelet neural network,it is the basis of multidimensional network, multidimensional neural network requires the use of direct product definition and multidimensional wavelet base, and use structure tensor product method. In wavelet neural network structure, a reasonable parameters selection for the entire network model has an important influence, when continuous wavelet neural use back propagation algorithm, its convergence is not ideal, and it easy to produce the problem such as optimization of oscillation and local extremum, therefore, we need to avoid this kind of situation.

Wavelet Quito resolution analysis: ulti-resolution analysis of wavelet base originated from S.M allat spatial structure theory. In wavelet neural network, the wavelet basis functions are not completely independent and there is a linear correlation between them, they can't satisfy all the orthogonality. This paper is based on multiresolution analysis method, wavelet orthogonal transformation can be achieved quickly build, this method make signal decomposition to the specified frequency band, it is a great significance for signal filtering.

The multi-resolution analysis of bearing fault signal is the essence of orthogonal wavelet filtering and decomposition. Fault signal under the action of high and low pass filter can get two sets of high and low different signals, each decomposition signals length is half its original signal, After processing the signal, every point sample can avoid redundant features, the quality of the signal is good. Because of the difference of bearing fault signal within the scope of the different energy, in order to facilitate signal processing, we can use scale method.

Multivariate time series analysis: This paper puts forward using multivariate time series analysis method to determine the input of neural network, thus can avoid the subjectivity of the human input, and ensure the accuracy and efficiency of the network. Multivariate time series analysis is the estimation of the network parameters calculation, It is able to maintain a lower error under correction model,it has a good application effect in the sample data processing of signal. Delay order of wavelet neural network model [5], can be used to check through the regression residual sequence algorithm ,the combination of multivariate time series analysis results and the model will eventually determine the appropriate network variable type and number. 


\section{Adjust parameters of wavelet neural network algorithm}

The output of the multidimensional wavelet neural network can be represented as:

$$
y=f\left(\sum_{i=1}^{k} w_{i} \psi_{i}\left(\left(\sum_{j=1}^{m} w_{i j} x_{j}-b_{i}\right) / a_{i}\right)-\theta\right)
$$

Acording to the formula, $m$ is the number of nodes in the input layer, $k$ is the number of nodes in the hidden layer, $x_{j}$ is the $j$ functions for network input nodes, $w_{i j}$ is the weights between the $i$ hidden node and the $j$ function input node, $\theta$ is the threshold for the output, $\psi_{i}$ is the role function, $a_{i}$ and $b_{i}$ are the scaling and translation parameters of hidden nodes respectively.Among them, the function role, bits of $\mathrm{I}$ in the form of the wavelet function can be represented as:

$$
\psi_{i}\left(\frac{x-b_{i}}{a_{i}}\right)=\cos \left(1.75 \frac{x-b_{i}}{a_{i}}\right) \exp \left(-0.5\left(\frac{x-b_{i}}{a_{i}}\right)^{2}\right)
$$

In order to improve the multidimensional wavelet neural network adaptive ability, In this paper, we revised sample parameters such as weights and thresholds, This method improved the operation efficiency of samples, Target error function of wavelet neural network are as follows:

$$
E=\sum_{p=1}^{P} E^{p}=\frac{1}{2 P} \sum_{p=1}^{P} \sum_{n=1}^{N}\left(d_{n}^{p}-y_{n}^{p}\right)
$$

Acording to the above formula, $d_{n}^{p}$ is a hidden layer of the Nth node expected output, $y_{n}^{p}$ is the actual output of neural network, $P(p=1,2, \cdots)$ is the output samples. In order to make the error function to achieve the minimum value, this paper defines the output of the hidden layer as $O_{k}^{p}$ :

$$
O_{k}^{p}=\mathrm{h}\left(\frac{I_{k}^{p}-b_{k}}{a_{k}}\right) \quad I_{k}^{p}=\sum_{m=1}^{M} w_{k m} X_{m}^{p}
$$

Acording to the above formula, $x_{m}^{p}$ is input values for the input layer, $w_{k m}$ is the weights between the number of nodes in the input layer $m$ and the hidden layer node number of $k$, the function of $h$ is Morlet wavelet function. The weights between input layer and hidden layer after adjustment can be represented as:

$$
\begin{aligned}
& w_{n k}^{\text {new }}=w_{n k}^{\text {old }}+\eta \sum_{m=1}^{p} \delta_{n k}+\lambda \Delta w_{n k}^{\text {old }} \\
& \delta_{n k}=\frac{\partial E_{n}^{p}}{\partial w_{n k}}=\left(d_{n}^{p}-y_{n}^{p}\right) \cdot y_{n}^{p} \cdot\left(1-y_{n}^{p}\right)
\end{aligned}
$$

Acording to the above formula, $w_{n k}^{\text {old }}$ and $w_{n k}^{\text {new }}$ is the correction weight pre-and post respectively, $\Delta w_{n k}^{\text {old }}$ is the momentum item, $\lambda$ is the momentum factor, $\eta$ is the learning efficiency, input layer and output layer have a same weight correction method. after adjusting the scaling parameters ,The wavelet function can be represented as:

$$
\begin{aligned}
& a_{k}^{\text {new }}=a_{k}^{\text {old }}+\eta \sum_{m=1}^{p} \delta_{a_{k}}+\lambda \Delta a_{k}^{\text {old }} \\
& \delta_{a_{k}}=\frac{\partial E_{n}^{p}}{\partial a_{k}}=\sum_{n=1}^{N}\left(\delta_{n k} w_{n k}\right) \cdot \frac{\partial O_{k}^{p}}{\partial a_{k}}
\end{aligned}
$$

\section{Numerical value simulation calculation}

In order to study the neural network algorithm based on wavelet pretreatment processing capacity of bearing fault waveform,and its parameter adjustment after the convergence [6], in this paper, using sample data, We conducted numerical simulation based on MATLAB, use the number of iterations and error diagram, verify the optimization effect of the parameter adjustment. The partial damage of bearing failure frequency (about $30 \mathrm{kHz}$ ) is low, This phenomenon leads to the resolution of the power spectrum, therefore, it need to reconstruction of wavelet coefficients in the numerical simulation, it need to use the theory of wavelet neural network identification, Finally we get the detection results of the analysis by using the Hilbert envelope demodulation method. 
By calculating, it is concluded that the bearing inner ring and outer ring fault waveform graph respectively as shown in Fig.2 and Fig.3, it can be seen in the figure, the waveform figure good resolution, The inner ring fault wave acceleration vibration frequency is lower and wave crest distribution is relatively sparse, the waveform is bigger than the outer ring wave amplitude.thus, neural network based on wavelet pretreatment technology for bearing fault signal has a good processing effect.

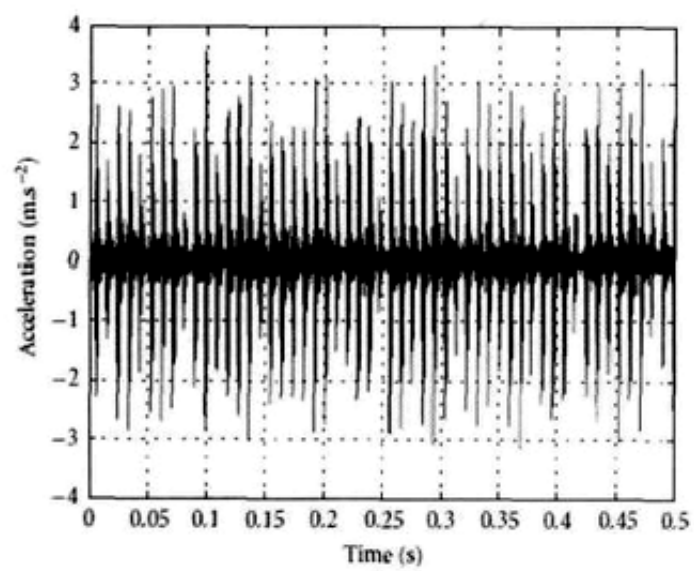

Fig.2 bearing inner ring fault waveform

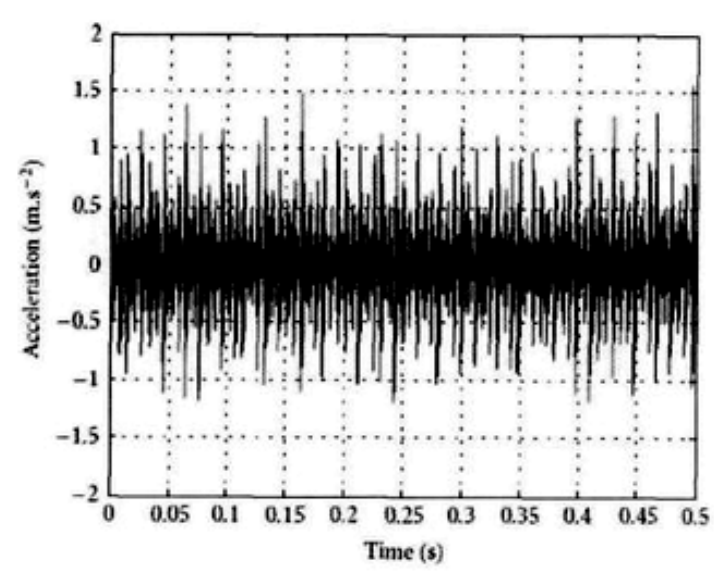

Fig.3 Bearing outer ring fault waveform

\section{Summary}

Preprocessing based on wavelet neural network technology in the bearing fault signal processing has a good application effect.The revised parameters of network structure in the signal processing has better convergence and lower error, it can reduce the blindness of neural network parameters design. although the multidimensional neural network theory is not mature enough, it has a broad prospect of application in signal processing, this memory has important significance.to improve the reliability of mechanical equipment, reduce the production cost.

\section{References}

[1] $\mathrm{Xu}$ Jinwu, $\mathrm{Xu} \mathrm{Ke}$. The application of wavelet transform in rolling bearing fault diagnosis [J]. Journal of mechanical engineering, 1997 (4) : 50-55.

[2] Wan Lianghong. Rolling bearing fault diagnosis method based on wavelet analysis to study [J]. Journal of bearing, 2004, 1:23-26.

[3] Zhang Guoyuan, Automatic monitoring and fault diagnosis system of rolling bearings [J]. Journal of bearing, 2005, 1:32-34.

[4] Zi Yang, Zhang Zhousuo. Wavelet analysis technology and its application in mechanical equipment nonstationary fault diagnosis [J]. Journal of xi 'an jiaotong university, 2000 (9) : 82-57.

[5] Li Jingyu, zhou, Li Qiqiang, Hou Haiyan,etc. Based on the genetic algorithm of wavelet neural network traffic flow prediction [J]. Journal of shandong university (engineering science), 2007 (2) : 109-112.

[6] Shan Haiou, Li Ping. The improvement and application of wavelet neural network [J]. Journal of gansu science, 2007, 12 (1) : 75-78. 